\title{
DESPLAZAMIENTO DE CONIDIAS DE Metarhizium anisopliae var. anisopliae EN COLUMNAS DE TRES SERIES DE SUELO
}

\author{
Displacement of conidia of Metarhizium anisopliae var. anisopliae in \\ columns of three soil series
}

\author{
Ana María Salazar P. ${ }^{1}$, Macarena Gerding G. ${ }^{2}$, Andrés France I. $^{*}$, Jorge Campos P. ${ }^{2}$, \\ Marcos Gerding P. ${ }^{1}$, Marco Sandoval E. ${ }^{2}$ y Viviana Becerra V. ${ }^{1}$
}

\begin{abstract}
A B S T R A C T
The fungus Metarhizium anisopliae isolation QuM270 is a promising control agent for the wheat white grub (Hylamorpha elegans Burm.), a Chilean scarabeid species whose larvae feed on roots of diverse crops and move in soil up to a depth of $25 \mathrm{~cm}$. In this study, the vertical movement of Qu-M270 conidias suspended in water was evaluated in three soil series from the Bío-Bío Region $\left(36^{\circ} 32^{\prime} \mathrm{S}\right.$ lat; $71^{\circ} 55^{\prime} \mathrm{W}$ long), Chile. Soil was obtained using PVC pipes 30 $\mathrm{cm}$ long by $20 \mathrm{~cm}$ in diameter. Conidia recovery was performed in a specific medium for Metarhizium anisopliae, by counting colony forming units in $1 \mathrm{~g}$ of dry soil. The Quella series (Aquic Durixerets) was more restrictive to the percolation of conidia, which were concentrated at the first $10 \mathrm{~cm}$. With the Arenales series (Dystric Xeropsamments) conidia were found below $15 \mathrm{~cm}$ and in percolated liquids. On the other hand, the Mirador series (Ultic Palexeralf) had a uniform distribution of conidia at every depth.
\end{abstract}

Key words: biological control, entomopathogenic fungus, infiltration, Hylamorpha elegans, biopesticide.

\section{R E S U M E N}

El hongo entomopatógeno Metarhizium anisopliae, aislamiento Qu-M270, es específico para el control del gusano blanco del trigo (Hylamorpha elegans Burm.), el cual se alimenta de raíces de diversos cultivos y se desplaza en el perfil del suelo hasta los 25 $\mathrm{cm}$ de profundidad. En este estudio se evaluó el desplazamiento vertical de conidias de Qu-M270, en suspensión acuosa, a través de columnas de suelo extraídas con tubos de $\mathrm{PVC}$ de $30 \mathrm{~cm}$ de largo y $20 \mathrm{~cm}$ de diámetro, en tres series de suelo de la Región del BíoBío ( $36^{\circ} 32^{\prime}$ lat. Sur; $71^{\circ} 55^{\prime}$ long. Oeste), Chile. La recuperación de conidias se realizó en medio específico para Metarhizium anisopliae, contabilizando unidades formadoras de colonias por gramo de suelo seco. La serie Quella (Aquic Durixerets) fue más restrictiva al paso de conidias, las que se concentraron en los primeros $10 \mathrm{~cm}$ de profundidad, a diferencia de la serie Arenales (Dystric Xeropsamments) en que se observó su presencia bajo los $15 \mathrm{~cm}$ y en los líquidos percolados. La serie Mirador (Ultic Palexeralf) presentó una distribución uniforme a lo largo de todo el perfil.

Palabras clave: control biológico, hongo entomopatógeno, infiltración, Hylamorpha elegans, biopesticida.

\footnotetext{
${ }^{1}$ Instituto de Investigaciones Agropecuarias, Centro Regional de Investigación Quilamapu, Casilla 426, Chillán, Chile.

E-mail: afrance@inia.cl *Autor para correspondencia.

${ }^{2}$ Universidad de Concepción, Facultad de Agronomía, Av. Vicente Méndez 595, Chillán, Chile.

Recibido: 5 de septiembre de 2006. Aceptado: 14 de diciembre de 2006.
} 


\section{INTRODUCCIÓN}

Dentro de las plagas de hábito subterráneo de importancia económica en Chile destaca la familia Scarabeidae cuyos estados larvales denominados "gusanos blancos" se encuentran asociados a praderas y cereales (Artigas, 1994) causando serios daños desde la Región del Maule ( $35^{\circ} 17^{\prime}$ lat. Sur; $71^{\circ} 17^{\prime}$ long. Oeste) al sur (Aguilera et al., 1996). Las especies más destacadas son Hylamorpha elegans (Burm.), Phytoloema hermanni(Germ.), y Sericoides germaini (Dalla Torre) (Norambuena y Aguilera, 1998), los cuales permanecen aproximadamente siete meses en estado larval. Durante ese tiempo las larvas cambian de profundidad de ubicación en el perfil del suelo, lo que es determinado en parte por la humedad del suelo, la temperatura y por la disponibilidad de raíces para alimento en estadios de mayor actividad alimentaria (Artigas, 1994).

En Chile se han seleccionado aislamientos de hongos entomopatógenos que han alcanzado un buen control de gusanos blancos. Se han logrado mortalidades de $H$. elegans cercanas al $95 \%$ bajo condiciones de laboratorio con la cepa nativa Qu-M270 de Metarhizium anisopliae, perteneciente a la colección de hongos entomopatógenos del Instituto de Investigaciones Agropecuarias (INIA) (Rodríguez et al., 2004).

Los hongos entomopatógenos se pueden aplicar de diversas formas, siendo la suspensión de conidias una de las más comunes y de mayor uso en el control de plagas subterráneas, ya que en el suelo las conidias están más protegidas de factores como la radiación ultravioleta (UV) y el viento, siendo capaces de desplazarse en el perfil hasta alcanzar la plaga a controlar (Storey y Gardner, 1988). Lo anterior está sujeto a diversos factores del suelo que pueden afectar la eficiencia y persistencia de los entomopatógenos, entre los cuales destacan el contenido de materia orgánica, la temperatura y humedad (Vänninen et al., 2000). En general, en condiciones naturales no existe una proliferación del inóculo en el suelo, sino que éste se mantiene en bajas densidades en las proximidades de su hospedero (McCoy et al., 2005).

El control microbiano de insectos surge como una alternativa interesante, ya que permite reducir la carga de químicos y la posibilidad que los insectos desarrollen resistencia. Por otra parte, cuando las conidias se aplican con agua son capaces de desplazarse en el perfil del suelo hasta alcanzar la plaga a controlar; sin embargo, se ha observado que esta capacidad está directamente influenciada por las características físicas de los suelos (Storey y Gardner, 1988). Dado lo anterior, este estudio tuvo por objetivo determinar la profundidad que son desplazadas las conidias de Metarhizium anisopliae por efecto del agua, en tres series de suelo, que representan el rango de estructuras presentes en la Región del Bío-Bío, Chile

\section{MATERIALES Y MÉTODOS}

\section{Extracción de las columnas de suelo}

Se utilizaron tres series de suelo presentes en la Región del Bío-Bío (36³2' lat. Sur; $71^{\circ} 55^{\prime}$ long. Oeste), Chile, las que se sometieron a un análisis físico (Laboratorio de Análisis de Suelos y Plantas de la Universidad de Concepción, Chillán) para determinar texturas y estructuras de los suelos (Cuadro 1). La muestra de suelo serie Quella (Aquic Durixererts), obtenida en el campo experimental de arroz (Oryza sativa L.) perteneciente al Instituto de Investigaciones Agropecuarias (INIA), Centro Regional de Investigación (CRI) Quilamapu, Chi-

Cuadro 1. Análisis físico de los suelos utilizados en el ensayo de desplazamiento de conidias. Chillán, Chile.

Table 1. Physical analysis of the soils used in the conidia displacement experiment. Chillán, Chile.

\begin{tabular}{|c|c|c|c|c|c|c|c|c|}
\hline Serie & $\begin{array}{c}\text { Arena } \\
(\%)\end{array}$ & $\begin{array}{c}\operatorname{Limo} \\
(\%)\end{array}$ & $\begin{array}{c}\text { Arcilla } \\
(\%)\end{array}$ & Textura $^{1}$ & Estructura $^{2}$ & $\begin{array}{l}\text { Densidad } \\
\text { aparente }\end{array}$ & $\begin{array}{c}\text { Macroporos } \\
(\%)\end{array}$ & $\begin{array}{c}\text { Microporos } \\
(\%)\end{array}$ \\
\hline Mirador & 44,1 & 21,2 & 34,7 & $\begin{array}{l}\text { Franco } \\
\text { arcillosa }\end{array}$ & $\begin{array}{c}\text { Bloques sub- } \\
\text { angulares }\end{array}$ & 1,21 & 24,04 & 30,3 \\
\hline Quella & 58,8 & 23,8 & 17,4 & $\begin{array}{l}\text { Franco } \\
\text { arcillosa }\end{array}$ & Masiva & 1,39 & 31,0 & 16,55 \\
\hline Arenales & 96,9 & 2,1 & 1,0 & Arenosa & Suelta & 1,30 & 42,74 & 8,2 \\
\hline
\end{tabular}

${ }^{1}$ Análisis de textura mediante el método del hidrómetro (Gee y Bauder, 1986).

${ }^{2}$ Análisis de densidad aparente mediante el método del terrón (Blake and Harte, 1986). 
llán ( $36^{\circ} 58^{\prime}$ lat. Sur; $72^{\circ} 08^{\prime}$ long. Oeste), correspondió a textura franco arcillosa con estructura masiva; la muestra de suelo serie Mirador (Ultic Palexeralfs) se obtuvo en el Campus Chillán de la Universidad de Concepción, Chillán ( $36^{\circ} 59^{\prime}$ lat. Sur; $72^{\circ} 08^{\prime}$ long. Oeste), con textura franco arcillosa y estructura de bloques sub-angulares; y por último la serie Arenales (Dystric Xeropsamments) presente en las cercanías de la ciudad de Cabrero (36 $96^{\circ}$ ' lat. Sur; $72^{\circ} 36^{\prime}$ long. Oeste) con textura arenosa y estructura suelta.

Para la extracción de cada núcleo de suelo se utilizaron tubos de policloruro de vinilo (PVC) de 20 $\mathrm{cm}$ de diámetro, $3 \mathrm{~mm}$ de espesor y $30 \mathrm{~cm}$ de longitud (Figura 1), en base a la profundidad a la cual pueden desplazarse las larvas de H. elegans en el perfil del suelo (Fresard, 1992).

Los tubos se introdujeron en cada perfil ejerciendo presión hasta enterrarlos completamente. Para cada tipo de suelo se extrajeron tres repeticiones. Además, se colectó una muestra control de $10 \mathrm{~g}$ a cada profundidad mediante barreno a las distintas profundidades en estudio $(0,5,10,15,20$ y $25 \mathrm{~cm})$, para corroborar la presencia natural de $M$. anisopliae en los suelos utilizados.

\section{Producción de inóculo}

El aislamiento de Metarhizium anisopliae cepa Qu-M270 utilizada para este ensayo, pertenece a la colección de hongos entomopatógenos del CRI Quilamapu, el cual se obtuvo a partir de una larva de H. elegans parasitada (France et al., 2000). El inóculo se obtuvo a partir de la cepa original mantenida en criopreservación en $\mathrm{N}$ líquido $\left(-196{ }^{\circ} \mathrm{C}\right)$, la cual se descongeló mediante un shock térmico por inmersión del criotubo en agua a $37{ }^{\circ} \mathrm{C}$, luego la suspensión de conidias se sembró con una asa en medio agar papa dextrosa (APD) (Becton, Dickinson and Company, Le Pont de Claix, France).

Para la multiplicación de conidias, se inoculó arroz precocido estéril en bolsas de polipropileno con un trozo de la colonia de Qu-M270 (Cherry et al., 1999). La cosecha de conidias se realizó mediante un tamizado con apertura de $100 \mu \mathrm{m}$, luego se deshidrataron y almacenaron al vacío (Rodríguez et al., 2004) hasta su utilización en el estudio.

Se realizó una prueba de germinación de conidias $24 \mathrm{~h}$ antes de la aplicación, la cual consistió en con- tabilizar el número de conidias germinadas mediante un microscopio compuesto de 100x (Leica, modelo DM/LS, Germany), desde una placa Petri con APD previamente inoculada con una alícuota de suspensión de agua más las conidias, e incubadas por $24 \mathrm{~h}$ en oscuridad y temperatura de $24 \pm 2{ }^{\circ} \mathrm{C}$. El porcentaje de viabilidad se obtuvo mediante la formula: número de conidias germinadas/número de conidias examinadas (Butt y Goettel, 2000). Una vez conocida la viabilidad de las conidias se preparó la dosis para cada bioensayo, contabilizando las conidias en cámara de recuento Neubauer (Boeco, Neubauer, Germany).

\section{Movimiento de conidias en núcleos de suelo}

En el laboratorio, los cilindros (Figura 1) fueron puestos sobre coladores plásticos y éstos sobre recipientes plásticos, de manera que el agua de saturación percolara a través de cada uno de los núcleos de suelo. En cada uno de los tubos se aplicó una suspensión acuosa de conidias de $M$. anisopliae cepa Qu-M270, en una concentración equivalente a $10^{13}$ conidias por hectárea (Rodríguez et al., 2004), lo que llevado al volumen del tubo y considerando $95 \%$ de viabilidad, significó una concentración de $9,8 \times 10^{7}$ conidias en $2,5 \mathrm{~mL}$ de agua destilada estéril. A la suspensión se le adicionó $0,05 \%$ de Tween-80 (Sigma, Steinheim, Germany) de manera de facilitar la suspensión de conidias (Gaugler et al., 1989).

Después de haber aplicado la suspensión concentrada de conidias, se utilizó agua destilada para saturar los suelos en dos oportunidades, en cada una de las series, con intervalos de $1 \mathrm{~h}$ luego de la primera saturación. Se consideró punto de saturación al momento en que percoló la primera gota de agua a través de los coladores, luego de lo cual se suspendió la aplicación de agua. Una vez saturados los suelos se dejaron drenar los tubos durante $24 \mathrm{~h}, \mathrm{y}$ el efluente caído a cada uno de los recipientes se almacenó a $5{ }^{\circ} \mathrm{C}$ (Storey y Gardner, 1987), para posteriormente observar la presencia o ausencia de conidias en los líquidos percolados. Transcurridas $24 \mathrm{~h}$, cada uno de los tubos se perforó utilizando un taladro con broca de paleta de $2,54 \mathrm{~cm}$ cada 5 $\mathrm{cm}$ a lo largo del tubo (Figura 1). A través de las perforaciones se obtuvo una muestra utilizando una espátula para cada profundidad $(0,5,10,15,20 \mathrm{y}$ $25 \mathrm{~cm}$ ), la que se depositó en tubos de ensayo. Posteriormente se pesó una submuestra de $1 \mathrm{~g}$ para cada tratamiento, la que se diluyó en $9 \mathrm{~mL}$ de agua des- 


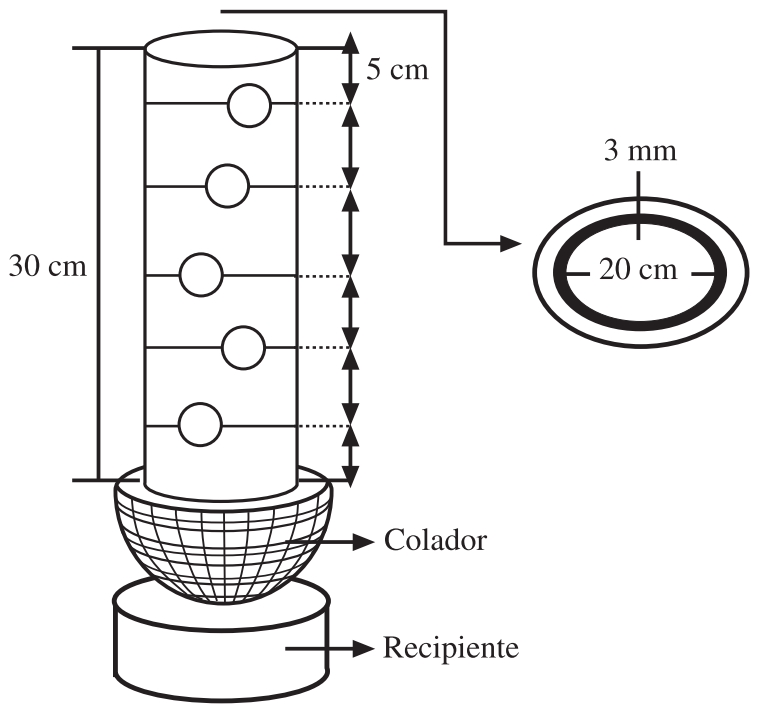

Figura 1. Esquema de la columna utilizada en ensayo de desplazamiento de conidias de Metarhizium anisopliae var. anisopliae (aislamiento Qu-M270).

Figure 1. Diagram of the column used to evaluate Metarhizium anisopliae var. anisopliae (isolate $\mathrm{Qu}-$ M270) conidia displacement.

tilada estéril dentro de un tubo de ensayo y se agitó mediante un vortex mecánico (Thermoline, modelo 37600, Dubuque, Iowa, USA). Además, en cada serie de suelo se tomó una muestra de $50 \mathrm{~g}$ para determinar el contenido de humedad.

Para comprobar la presencia o ausencia del hongo a distintas profundidades y texturas de suelo, se extrajo $0,1 \mathrm{~mL}$ de suspensión, utilizando una micropipeta de $1000 \mu \mathrm{L}$ (Socorex, Swiss), alícuota que se sembró sobre placas Petri con medio selectivo para M. anisopliae (Chase et al., 1986) y se esparció con una bagueta de vidrio, de manera de distribuir la suspensión sobre toda la placa. Las placas sembradas se incubaron durante 30 días en una cámara acondicionada a $25{ }^{\circ} \mathrm{C}$ (Alves, 1998; Hallsworth y Magan, 1999) y oscuridad (Bidochka et al., 1998). Se observó y registró la aparición de colonias de $M$. anisopliae sobre las placas, las que se reconocieron en lupa estereoscópica por la formación de un micelio blanco y denso, y un anillo de conidias verde oliva (Chase et al., 1986).

El diseño experimental fue completamente al azar con 18 tratamientos, correspondientes a la combi- nación de las diferentes series y profundidades de suelo, con tres repeticiones. Los resultados se expresaron para cada serie y profundidad en unidades formadoras de colonias (UFC) $\mathrm{g}^{-1}$ de suelo seco (Miles y Misra, 1938). Estos datos se transformaron por medio de $(x+1)^{1 / 2}$, y luego se analizaron mediante un análisis de varianza (ANDEVA) (Steel y Torrie, 1988). Posteriormente se realizó la comparación de medias mediante el test de Tukey $(\mathrm{P} \leq$ $0,05)$ (Steel y Torrie, 1988), con el software estadístico InfoStat (2004) de la Universidad Nacional de Córdoba, Argentina. Además se realizó un análisis de regresión no lineal (InfoStat, 2004) para modelar el comportamiento de las conidias bajo diferentes profundidades y series de suelo, para obtener la profundidad en la cual se concentró la mayor cantidad de conidias durante el ensayo mediante la derivada del modelo e igualando la ecuación a 0 .

\section{RESULTADOS Y DISCUSIÓN}

\section{Desplazamiento de conidias en las columnas de suelo}

En las muestras de suelo control no se detectaron propágalos de $M$. anisopliae, con lo cual los siguientes resultados se atribuyen al inóculo que se agregó a los diferentes tratamientos. En suelo de la serie Quella el hongo fue recuperado en los primeros $10 \mathrm{~cm}$ de profundidad, no encontrándose esporas a mayor profundidad ni en el agua percolada. Se observó diferencias $(\mathrm{P} \leq 0,05)$ a los $0 ; 5 ; \mathrm{y} 10 \mathrm{~cm}$ de profundidad con respecto a la serie Arenales (Cuadro 2), donde se produjo una ausencia total de conidias en las profundidades antes mencionadas. Los suelos de textura arenosa poseen un alto contenido de macroporos, facilitando de esta manera el movimiento del aire y agua de drenaje (Foth, 1990), lo que explicaría la mayor penetración vertical de las conidias hacia capas más profundas, ya que se concentraron bajo los $15 \mathrm{~cm}$.

En contraste, la muestra de suelo serie Quella posee una capacidad de infiltración mucho menor debido a la baja presencia de macroporos y mayor presencia de microporos (Storey y Gardner, 1988), lo que explica la presencia de conidias sólo en los primeros $10 \mathrm{~cm}$. Además, la cantidad de UFC fue inferior al total de conidias aplicadas, lo que se podría atribuir a esta lenta infiltración que incrementa el tiempo de las conidias en suspensión acuosa, y en consecuencia permite la germinación antes 
de ingresar al suelo, con la consiguiente reducción en la viabilidad y persistencia por falta de oxígeno (Storey y Gardner, 1987; Moore et al., 1997).

Por otro lado, Ignoffo et al. (1997) concluyeron que las partículas de arcilla tienen la capacidad de adsorber conidias, como las del hongo entomopatógeno Nomuraea rileyi, produciendo la retención de éstas en las capas superficiales, lo que explica en parte lo sucedido en la serie Quella. Aunque esto se contrapone con lo observado por Storey et al. (1989), quienes concluyeron que el alto contenido de arcilla no restringió el movimiento vertical de conidias del hongo entomopatógeno Beauveria bassiana. Lo anterior concuerda con lo observado en la serie Mirador, ya que a pesar del alto contenido de arcilla, incluso superior al de la serie Quella, las UFC fueron recuperadas en forma homogénea en todas las profundidades. La concentración de conidias fue similar a la serie Quella a los 0; 5 y $10 \mathrm{~cm}$.

En las profundidades de $15 ; 20$; y $25 \mathrm{~cm}$ se presentaron diferencias entre los suelos serie Mirador y Quella, ya que en este último no hubo presencia de UFC en ninguna de las profundidades mencionadas. A pesar de poseer la misma textura, la presencia de colonias en la serie Mirador fue similar en las tres profundidades, lo cual se debería principalmente a la estructura de ambos suelos, siendo masiva para Quella y de bloques subangulares para Mirador (Cuadro 1), permitiendo a este último un mejor drenaje y

Cuadro 2. Unidades formadoras de colonias (UFC) de Metarhizium anisopliae var. anisopliae aislamiento Qu-M270 en diferentes series y profundidades de suelo.

Table 2. Metarhizium anisopliae var. anisopliae isolate Qu-M270 colony forming units (CFU) in different series and soil depths.

\begin{tabular}{cccc}
\hline $\begin{array}{c}\text { Profundidad } \\
(\mathbf{c m})\end{array}$ & \multicolumn{3}{c}{ UFC $\left(\mathbf{x} \mathbf{1 0}^{\mathbf{2}}\right) \mathbf{g}^{-1}$ de suelo seco } \\
\cline { 2 - 4 } & $\begin{array}{c}\text { Serie } \\
\text { Arenales }\end{array}$ & $\begin{array}{c}\text { Serie } \\
\text { Mirador }\end{array}$ & $\begin{array}{c}\text { Serie } \\
\text { Quella }\end{array}$ \\
\hline 0 & $0,0 \mathrm{~b}^{*}$ & $6,6 \mathrm{a}$ & $4,4 \mathrm{a}$ \\
5 & $0,0 \mathrm{~b}$ & $9,3 \mathrm{a}$ & $8,4 \mathrm{a}$ \\
10 & $0,0 \mathrm{~b}$ & $8,8 \mathrm{a}$ & $9,2 \mathrm{a}$ \\
15 & $0,7 \mathrm{ab}$ & $8,4 \mathrm{a}$ & $0,0 \mathrm{~b}$ \\
20 & $0,7 \mathrm{~b}$ & $6,6 \mathrm{a}$ & $0,0 \mathrm{~b}$ \\
25 & $1,1 \mathrm{~b}$ & $5,7 \mathrm{a}$ & $0,0 \mathrm{~b}$ \\
\hline
\end{tabular}

* Letras distintas en cada fila indican diferencias estadísticas entre tratamientos de acuerdo al test de Tukey $(\mathrm{P} \leq 0,05)$. un buen comportamiento frente a aplicaciones de formulaciones de conidias en suspensión acuosa. Esta mayor infiltración en la serie Mirador se podría explicar por la probable presencia de macroporos y microporos en cantidades similares, lo que ayuda a una mejor infiltración de agua a lo largo de todo el perfil, beneficiando con esto al movimiento de conidias a las diferentes profundidades.

En la serie Arenales se verificó la presencia de conidias en las tres últimas profundidades, pero no hubo diferencias con respecto a la serie Quella. La cantidad de UFC recuperadas a esas profundidades fue mucho menor con respecto a las conidias aplicadas, las cuales percolaron masivamente hacia el final del tubo. En este tipo de suelo la carga de agua debiera ser manejada para llegar a la plaga que se desea controlar. En el caso de plagas superficiales, la carga de agua debería ser mucho menor, aunque la falta de humedad en las conidias afecta su longevidad y persistencia en el suelo (Storey y Gardner, 1988).

Estudios en diferentes suelos indicarían que la textura y materia orgánica son los principales mecanismos que influyen en el movimiento vertical de conidias en suspensión acuosa, siendo los suelos arenosos y bajos en materia orgánica los que retendrían menores cantidades de conidias, que aquellos arcillosos y orgánicos (Ignoffo, et al., 1977; Storey and Gardner, 1988). Los mecanismos responsables de la retención de conidias no son del todo conocidos, pero estarían relacionados con la capacidad de intercambio catiónico y el tamaño de los poros. Además, el movimiento vertical de los propágulos también depende de las características propias de éstos, tales como el tamaño (Inglis, et al., 2001).

El análisis de regresión no lineal de las conidias bajo diferentes profundidades y series de suelo (Figura 2), permitió predecir mediante la derivada de la ecuación a qué profundidad se encontró la mayor concentración de conidias. Para suelos de la serie Arenales, la mayor concentración se presentó a los $26 \mathrm{~cm}$, lo cual demuestra que la mayor cantidad de inóculo quedó en los líquidos percolados; por consiguiente, formulaciones de agua más conidias pueden ser lixiviadas con facilidad en suelos de alta infiltración. Vänninen et al. (2000) realizaron ensayos de persistencia con el hongo entomopatógeno $M$. anisopliae bajo diferentes texturas, $\mathrm{y}$ encontraron que en suelos con mayor contenido de 
arena el movimiento de las conidias fue mayor, haciéndose necesario incrementar la eficiencia del hongo mediante formulaciones granulares, que permiten disminuir la lixiviación de conidias durante lluvias fuertes o altas cargas de agua (Bustillo et al., 1999).

En los núcleos de suelo de la serie Quella la mayor concentración de esporas se registró a los $6 \mathrm{~cm}$ de profundidad. En suelos de la serie Mirador la ma- yor presencia de conidias se presentó a los $10 \mathrm{~cm}$, aunque se observa que a mayores profundidades hubo presencia de conidias (Figura 2). En suelos de esta serie, $M$. anisopliae podría ser aplicado en suspensión de conidias en agua, ya que es posible que el inóculo penetre y llegue al lugar donde se encuentran las larvas, como se apreció en este ensayo, donde el inóculo penetró $25 \mathrm{~cm}$, que corresponde a la máxima profundidad que pueden alcanzar las larvas de H. elegans (Fresard, 1992).
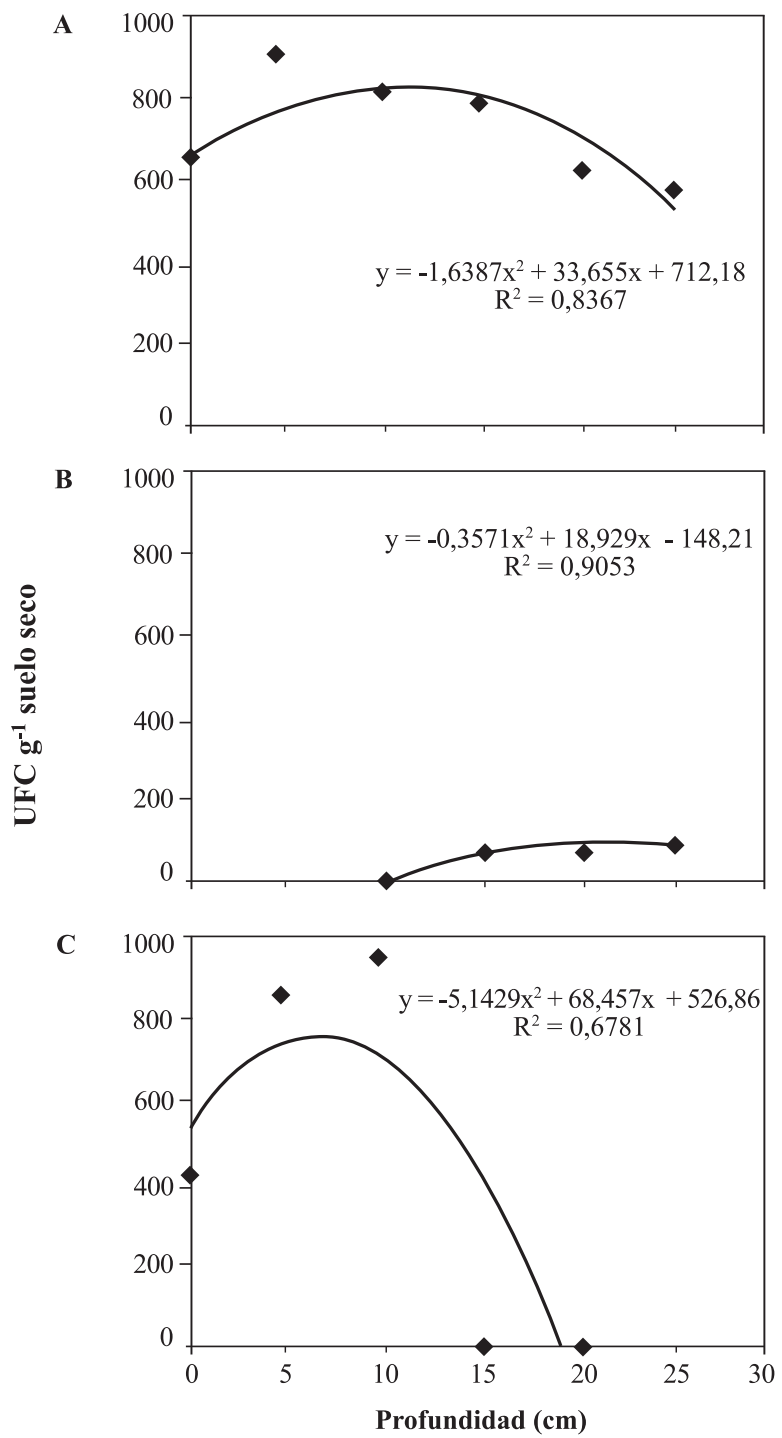

Figura 2. Modelo del desplazamiento de las conidias de Metarhizium anisopliae var. anisopliae aislamiento Qu-M270 en diferentes profundidades y series de suelo: A: Mirador; B: Arenales y C: Quella.

Figure 2. Model of displacement of Metarhizium anisopliae var. anisopliae isolate Qu-M270 conidia at different soil depths and soil series: A: Mirador; B: Arenales and C: Quella. 


\section{CONCLUSIONES}

Las conidias de hongos entomopatógenos, como Metarhizium anisopliae var. anisopliae, pueden desplazarse verticalmente en los perfiles de suelo con ayuda del agua. El grado de infiltración que pueden alcanzar depende de la estructura del suelo, de manera que en la serie Quella la mayor concentración de conidias se registró a los $6 \mathrm{~cm}$ de profundidad, en la serie Arenales presentó una mayor concentración de conidias bajo los $25 \mathrm{~cm}$, y en suelos francos, como la serie Mirador, se logró una distri- bución homogénea de conidias hasta las profundidades que alcanzó el nivel de agua infiltrada.

\section{RECONOCIMIENTOS}

Esta investigación fue financiada por el Fondo Nacional de Investigación Científica y Tecnológica, FONDECYT ( ${ }^{\circ}$ 1030037). Se agradece al Laboratorio de Análisis de Suelos y Plantas de la Facultad de Agronomía de la Universidad de Concepción, por el análisis físico de los suelos utilizados, en especial al Sr. Manuel Zapata.

\section{LITERATURA CITADA}

Aguilera, A., E. Cisternas, M. Gerding, y H. Norambuena. 1996. Plagas de las praderas. p. 309-340. In I. Ruiz (ed.). Praderas para Chile. $2^{\text {a }}$ ed. Instituto de Investigaciones Agropecuarias, Santiago, Chile.

Alves, S.B. 1998. Fungos entomopatogenicos. p. 289-370. In S. B. Alves (ed.). Controle microbiano de insectos. $2^{\mathrm{a}}$ ed. Fundación de Estudios Agrarios Luiz de Queiroz, Piracicaba, São Paulo, Brasil.

Artigas, J. 1994. Entomología económica. Insectos de interés agrícola, forestal, médico y veterinario. Vol. II. 943 p. Ediciones Universidad de Concepción, Concepción, Chile.

Bidochka, M., J. Kasperski, and G.A. Wild. 1998. Ocurrence of entomopathogenic fungi Metarhizium anisopliae and Beauveria bassiana in soils from temperate and near-northern habitat. Can. J. Bot.76:1198-1204.

Blake, G.R. and K.H. Harte. 1986. Bulk density. p. 371373. In A. Klute (ed.). Methods of soil analisis. Part 1. Physical and mineralogical (2 ed.). American Society of Agronomy, Madison, Wisconsin, USA.

Bustillo, A., M. Bernal, P. Benavides, and B. Chaves. 1999. Dymanics of Beauveria bassiana and Metarhizium anisopliae infecting Hypothenemus hampei (Coleoptera: Scolytidae) populations emerging from fallen coffee berries. Fla. Entomol. 82:491-497.

Butt, T., and M.S. Goettel. 2000. Bioassays of entomogenous fungi. p. 141-195. In A. Navon y K.R. Ascher (eds.). Bioassays of entomopathogenic microbes and nematodes. CAB International, Wallingford, UK.

Chase, A.R., L.S. Osborne, and V.M. Ferguson. 1986. Selective isolation of the entomopathogenic fungi Beauveria bassiana and Metarhizium anisopliae from an artificial potting medium. Fla. Entomol. 69:285-292.
Cherry, A., L. Jenkings., G. Heviefo., R. Bateman and C. Lomer. 1999. Operational and economic analysis of a West African pilot-scale production plant for aerial conidia of Metarhizium spp. for use as a mycoinsecticide against locust and grasshoppers. Biocontrol Science and Technology 9:35-51.

Foth, H. 1990. Fundamentals of soil science. p. 34-36. $8^{\text {th }}$ ed. John Wiley and Sons, New York, USA.

France, A., M. Gerding G., M. Gerding P., y A. Sandoval. 2000. Patogenicidad de una colección de cepas nativas de Metarhizium spp. y Beauveria spp. en Aegorhinus superciliosus, Asynonychus cervinus y Otiorhynchus sulcatus. Agric. Téc. (Chile) 60:205-215.

Fresard, M.E. 1992. Aspectos biológicos de Hylamorpha elegans Burmeister y fitofagia de larvas de dos especies de Scarabeidos en plantas de trigo. $236 \mathrm{p}$. Tesis Ingeniero Agrónomo. Universidad Austral, Facultad de Ciencias Agrarias, Valdivia, Chile.

Gaugler, R., S.D. Costa, and J. Lashomb. 1989. Stability and efficacy of Beauveria bassiana soil inoculations. Environ. Entomol. 18:412-417.

Gee, G.W., and J. W. Bauder. 1986. Particle size analysis. p. 404-408. In A. Klute (ed.). Methods of soil analysis. Part 1. Physical and mineralogical (2nd ed.) American Society of Agronomy, Madison, Wisconsin, USA.

Hallsworth, J.E., and N. Magan. 1999. Water and temperature relations of growth of entomopathogenous fungi Beauveria bassiana, Metarhizium anisopliae and Paecilomyces farinosus. J. Invertebr. Pathol. 74:261-266.

Ignoffo, C.M., C. García, L. Hostetter, and R.E. Pinnel. 1997. Vertical movement of conidia of Nomuraea rileyi through sand and loam soils. J. Econ. Entomol. 70:163-164. 
InfoStat. 2004. Manual de usuario. Actualizaciones. Versión 2004. Grupo InfoStat, Universidad de Córdoba, FCA. Editorial Brujas, Buenos Aires, Argentina.

Inglis, G., M. Goettel, T. Butt and H. Strasser. 2001. Use of Hyphomycetous fungi for managing insect pests. p. 23-69. In T.M. Butt, C.W. Jackson and N. Magan. Fungi as biocontrol agents. Progress, problems and potencial. CABI Internacional, Wallingford, UK.

McCoy, C., E.D. Quintela, and M. de Faria. 2005. Environmental persistence of entomopathogenic fungi. Luisiana State University Agricultural Center, Baton Rouge, Luisiana, USA. Available at http:// www.agctr.Isu.edu/s265/mccoy.htm Accessed 30 August 2005

Miles, A.A., and S.S. Misra. 1938. The estimation of the bactericidal power of blood. J. Hygiene 38:732-749.

Moore, D., J. Langewald, and F. Obognon. 1997. Effects of rehydration on the conidial viability of Metarhizium flavoviride mycopesticide formulations. Biocontrol Sci. Technol. 7:87-94.

Norambuena, H., y A. Aguilera. 1998. Plagas de las praderas. p. 229-248. In I. Ruiz (ed.). Praderas para Chile. Instituto de Investigaciones Agropecuarias, Santiago, Chile.
Rodríguez, M.E., A. France, y M. Gerding. 2004. Evaluación de dos cepas nativas del hongo Metarhizium anisopliae var. anisopliae (Metsh) para el control de larvas de gusano blanco Hylamorpha elegans Burm. (Coleoptera: Scarabaeidae). Agric. Téc. (Chile) 64:17-24.

Steel, R.G., y J.H. Torrie. 1988. Bioestadística: Principios y procedimientos. $622 \mathrm{p} .2^{\mathrm{a}}$ ed. McGraw-Hill, México D.F.

Storey, G., and W.A. Gardner. 1987. Vertical movement of commercially formulated Beauveria bassiana conidia through four Georgia soil types. Environ. Entomol. 16:178-181.

Storey, G., and WA. Gardner. 1988. Movement of an aqueous spray of Beauveria bassiana into the profile of four Georgia soils. Environ. Entomol. 17:135-139.

Storey, G., W.A. Gardner, and E.W. Tollner. 1989. Penetration and persistence of commercially formulated Beauveria bassiana conidia in soil of two tillage systems. Environ. Entomol. 18:835-839.

Vänninen, I., J. Tyni-Juslin, and H. Hokkanen. 2000. Persistence of augmented Metarhizium anisopliae and Beauveria bassiana in Finnish agricultural soils. BioControl 45:201-222. 